\title{
Parenteral lipid emulsions and phagocytic systems
}

\author{
D. L. Waitzberg ${ }^{1}$, P. H. Lotierzo ${ }^{1}$, A. F. Logullo $^{1}$, R. S. M. Torrinhas ${ }^{1}$, \\ C. C. A. Pereira ${ }^{1}$ and Rémy Meier ${ }^{2}$ \\ ${ }^{1}$ Departamento de Gastroenterologia, Faculdade de Medicina da Universidade de São Paulo, \\ FMUSP, Av. Dr Arnaldo 455- $3^{\circ}$ andar, CEP: 01246-903, São Paulo, Brazil \\ ${ }^{2}$ Department of Gastroenterology, University Hospital, Liestal, Switzerland
}

\begin{abstract}
Lipid emulsions (LE) for parenteral use are complex emulsions containing fatty acids, glycerol, phospholipids and tocopherol in variable amounts and concentrations. In clinical practice, LE have been employed for more than 30 years. Fatty acids may have different impacts on phagocytic cells according to their structure. Experimental and clinical studies have consistently shown that LE modify monocyte/macrophage and polymorphonuclear phagocytosis. The inhibitory effect of LE on the functional activity of the phagocytic system, although still clinically controversial, may have a harmful impact because total parenteral nutrition with lipids may be recommended in hypercatabolic conditions where inflammation and infection are present. LE based on triglycerides containing long chain fatty acids (termed long chain triglycerides or LCT) are the main parenteral fat source and are typically rich in $n-6$ polyunsaturated fatty acids. They may have adverse effects on the immune system, especially when given in high doses over a short period of time. However when administered properly they can be used safely. LE containing medium chain triglycerides (MCT) may have some advantages because of their positive effects on polymorphonuclear cells, macrophages, and cytokine production, particularly in critically ill or immunocompromized patients. New parenteral LE containing $n-3$ polyunsaturated fatty acids or monounsaturated olive oil are already available in Europe. Judicious use of these new LE is mandatory especially relating on their potential impact on the immune system. New experimental and clinical studies are required to further establish the role of LE in clinical nutrition.
\end{abstract}

Parenteral nutrition: Macrophage: Lipid: Neutrophil: Phagocytosis

\section{General considerations}

Indian scriptures dating back to $5000 \mathrm{BC}$ have documented that nutritional status has been associated with health (Chandra, 1985). Nutritional depletion results in immunosuppression, and therefore host defense impairment, favoring increased infection and mortality rates (Mullen et al. 1980). Improvement of nutritional state with nutritional interventions may re-establish immune competence and decrease the frequency and severity of infectious complications in hospitalized patients (Dudrick et al. 1969). Total parenteral nutrition (TPN) is one well-established nutritional therapy, which allows intravenous feeding of patients without an available enteral route (Koss et al. 1979). TPN is widely applied in hospital settings and consists of a solution containing amino acids, glucose, electrolytes and vitamins as well as lipid emulsions (LE). In
TPN regimens, LE function as calorie dense nutrients and a source of essential fatty acids.

Fatty acids (FA) are the main component of cell membranes. They are responsible for membrane structural integrity and the production of eicosanoids. Lipids are also major metabolic regulators and can modulate the immune response (Meade \& Mertin, 1978; DeWille et al. 1979; Kinsella, 1990).

Research on the effects of fatty acids on the immune response date back for over 30 years (Piette \& Saugier, 1970; Mertin \& Meade, 1977). The impact of intravenously infused isolated fatty acids on the immune system has been studied since 1972 (Di Luzio, 1972). It is well known that changes in the quality and amount of dietary fat (Hwang, 1989), as well as abnormalities in lipid metabolism (Koss et al. 1979; Beisel, 1981) can alter the immune response. There is much evidence that LE also have an impact on

\footnotetext{
Abbreviations: LE, lipid emulsions; TPN, total parenteral nutrition; LCT, long chain triglycerides; MCT, medium chain triglycerides; FA, fatty acids.

* Corresponding author: Prof. D. L. Waitzberg, fax 55 (11) 2856512, email d.waitzberg@terra.com.br
} 
mononuclear phagocytic function, particulary phagocytosis (Strunk et al. 1979; Wiernik et al. 1983; Cleary \& Pickering, 1983; Waitzberg et al. 1996). The purpose of this review is to examine the effects of different LE on the phagocytic process of macrophages and polymorphonuclear cells.

\section{Lipid emulsions}

LE are the parenteral source of essential fatty acids. They can be infused alone or together with glucose and amino acids, in malnourished or hypercatabolic patients. LE are triglyceride droplets enveloped with a stabilizing superficial layer of phospholipids (Shils et al. 1998) and their formulation includes substances other than triglycerides, such as lecithin, glycerol and alpha-tocopherol in variable amounts. The triglycerides may be presented structurally as long chain triglycerides (LCT) or medium chain triglycerides (MCT). LCT LE have been used in clinical practice for over 30 years. LCT contain fatty acid chains with 14, 16, 18, 20 and 22 carbon atoms and sometimes with double bonds. The number of double bonds present defines the fatty acids in LCT as saturated, mono or polyunsaturated. The double bonds can be found in different places in the carbon chain, their position follows rules governed by the specificity of the enzymes responsible for inserting them. If the first double bond is on carbon number 3, 6 or 9 from the methyl end of the carbon chain then the fatty acid is $n-3, n-6$ or $n-9$, respectively. LE which are commercially available for parenteral use are based on LCT containing a high proportion of $n-6$ polyunsaturated fatty acids usually derived from soybean or safflower oil, although LCT containing $n-3$ polyunsaturated fatty acids, derived from fish oil, and n-9 monounsaturated fatty acids from olive oil are also available. Saturated fatty acids with chains containing $6,8,10$ or 12-carbon atoms form the MCT emulsions. MCT are found in high amounts in coconut oil. For clinical purposes MCT is used as a $50 \%$ physical or structured mixture with LCT. Thus MCT/LCT LE have a lower proportion of LCT volume/volume than LCT LE.

Recently in Europe LE containing LCT or LCT/MCT enriched with fish oil (FO) became available for research. FO includes long chain triglycerides with twenty or more carbon atoms where the first double bond is located between the third and the fourth carbons from the methyl terminal of the fatty acids chain (omega-3 or $n-3$ ).

The incorporation of $n-3$ fatty acids into macrophage membranes occurs in 3-6 hours in vitro and in 3 days in vivo. The incorporation of different fatty acids may change membrane fluidity, alter phosphatidylinositol production and dienoic eicosanoid secretion and modulate the generation of interleukins and tumour necrosis factor (TNF) in response to lipopolysaccharide (LPS) stimulation. These effects are related to the membrane $n-6: n-3$ fatty acid ratio and to the total amount of $n-3$ and $n-6$ FA infused.

The physicochemical nature of the infused triacylglycerol (i.e. carbon chain length, saturation degree, size of the particle and nature of fatty acid) may determine structural changes and alterations in the phagocytic cell activity.

\section{Effects of lipid emulsions on monocyte-macrophages}

Monocyte-macrophages are immune cells involved in phagocytosis, antigen presentation and cytokine production. A number of experimental and clinical studies investigating the effects of currently used LE on monocyte-macrophage functions are summarized in Table 1.

\section{Experimental studies}

Experimental studies regarding the effects of LE on phagocytosis by monocyte-macrophages are controversial. Several trials have used rats, guinea pigs, and mice infused with different LE alone or as a component of TPN and studied the effects on circulating and resident macrophages.

Experimental lipid-TPN infusion for a week has been associated with decreased phagocytosis and increased superoxide production by peritoneal macrophages, splenic macrophage proliferation and bacterial translocation (Shou et al. 1994). Intravenous infusion of LCT LE and MCT/LCT LE into rats infected by Escherichia coli (E. coli) was followed by hyperplasia of Kupffer cells and splenic histiocytes (Waitzberg et al. 1992). The infusion of TPN with LCT LE into guinea pigs reduced phagocytosis of $E$. coli by Kupffer cells and splenic macrophages (Hamawy et al. 1985).

TPN with LCT LE was associated with inhibition of pulmonary bacterial phagocytosis but this was not observed with TPN with MCT/LCT (Sobrado et al. 1985). These changes were not verified in a similar experimental model in septic rats infused with TPN containing $50 \%$ non-protein calories in the form of LCT LE (Vallgren et al. 1986).

Despite reports indicating inhibitory effects of LCT LE, including anatomical-pathological alterations of monocytes and macrophages (Strunk et al. 1979; Nugent, 1984), other evaluations of the effect of LCT and MCT/LCT LE infusions in animals showed no alterations of phagocytosis and other monocyte/macrophage functions at different doses and periods and rates of infusion (Nishiwaki et al. 1986; Allen \& Murray, 1986; Waitzberg et al. 1996). Moreover it seems that there are distinct macrophage responses to intravenous infusion of LE according to the anatomical origin of macrophages. The total number of liver and spleen macrophages were reduced but pulmonary phagocytosis was increased when TPN enriched with LCT LE was compared to an oral diet (Cukier et al. 1999). These differences between studies may be attributed to differences in the experimental models used, and the amount of lipids administered (Table 1).

The addition of a fish oil LE to LCT and MCT/LCT LE increased total liver and lung macrophage number and phagocytosis in rats after $96 \mathrm{~h}$ of intravenous infusion (Cukier et al. 1999).

\section{Human studies}

Not many human clinical studies have investigated the effects of LE on monocyte function. Wiernik et al. (1983) infused $20 \%$ soybean oil emulsion intravenously for $2 \mathrm{~h}$ into healthy volunteers. During the infusion a significant increase in nitroblue tetrazolium-reduction by blood 
Table 1. Studies of lipid emulsions on monocyte/macrophage function

\begin{tabular}{|c|c|c|c|c|c|c|c|}
\hline Reference & Study in & Type of LE & Via & Dose & Time $(h)$ & Function & Findings \\
\hline \multirow[t]{2}{*}{ Waitzberg et al. 1997} & $\begin{array}{l}\text { Gastric cancer } \\
\text { patients } \\
\text { (10) }(+ \text { TPN) }\end{array}$ & LCT $10 \%$ & i.v. & $\begin{array}{l}10 \mathrm{kcal} / \mathrm{kg} \text { per day } \\
(0.08 \mathrm{~g} / \mathrm{kg} \mathrm{per} \mathrm{h})\end{array}$ & 48 & Chemotaxis & No alteration \\
\hline & & MCT/LCT $10 \%$ & & & & $\begin{array}{l}\text { Bacterial killing } \\
\text { Phagocytosis }\end{array}$ & $\begin{array}{l}\text { No alteration } \\
\text { No alteration }\end{array}$ \\
\hline Wiernik et al. 1983 & Healthy volunteers (18) & LCT $20 \%$ & i.v. & $20-100 \mathrm{mg} / \mathrm{ml}$ & 0.5 & NBT reduction & $\uparrow$ \\
\hline Strunk et al. 1979 & Guinea pigs & LCT $10 \%$ & i.v. & $93-375 \mathrm{mg} / \mathrm{l}$ & 48 & $\begin{array}{l}\text { Ability of peritoneal } \mathrm{M} \phi \text { to spread } \\
\text { Number of membrane ruffles } \\
\text { Complexity of membrane ruffles } \\
\text { Phagocytosis }\end{array}$ & $\begin{array}{l}\downarrow \\
\downarrow \\
\downarrow \\
\downarrow\end{array}$ \\
\hline Nugent, 1984 & Mice & LCT & i.v. & $40 \%$ vol & 18 & $\begin{array}{l}\text { Appearance of peritoneal } \mathrm{M} \phi \\
\text { Phagocytosis } \\
\text { Pinocytosis }\end{array}$ & $\begin{array}{l}\text { Rounded and more dense } \\
\downarrow \\
\downarrow\end{array}$ \\
\hline Allen \& Murray, 1986 & Mice & LCT & i.v. & $2 \mathrm{~g} / \mathrm{kg}$ per $\mathrm{d}$ & 240 & $\begin{array}{l}\text { PHI } \\
\text { Liver and spleen size }\end{array}$ & $\begin{array}{l}\text { No alteration } \\
\text { No alteration }\end{array}$ \\
\hline Nishiwaki et al. 1986 & Septic rats & LCT & i.v. & $25 \%$ of NPC & 72 & Phagocytosis & No alteration \\
\hline \multirow[t]{2}{*}{ Waitzberg et al. 1992} & $\begin{array}{l}\text { Rats with } E . \text { coli } \\
\text { peritonitis }\end{array}$ & LCT $10 \%$ & i.v. & $5 \mathrm{ml} / \mathrm{kg}$ per $\mathrm{h}$ & 44 & Chemotaxis & No alteration \\
\hline & & $\begin{array}{l}\text { MCT/LCT 10\% } \\
\text { MCT } 10 \%\end{array}$ & & & & $\begin{array}{l}\text { Phagocytosis } \\
\text { Bacterial killing }\end{array}$ & $\begin{array}{l}\text { No alteration } \\
\text { No alteration }\end{array}$ \\
\hline \multirow[t]{2}{*}{ Cukier et al. 1999} & Rats & LCT $10 \%$ & i.v. & $\begin{array}{l}2 \mathrm{ml} / \mathrm{kg} \text { per } \mathrm{h} \\
\text { ( } 31 \% \text { of } \mathrm{NPC})\end{array}$ & 96 & $\mathrm{PHI}$ of liver $\mathrm{M} \phi$ & $\uparrow$ \\
\hline & & $\begin{array}{l}\text { LCT + FO (9:1 vol/vol) } \\
\text { LCT + FO (1:1 vol/vol) } \\
\text { MCT/LCT + FO } \\
\text { (9:1 vol/vol) }\end{array}$ & & & & $\begin{array}{l}\mathrm{PHI} \text { of liver and lung } \mathrm{M} \phi \\
\mathrm{PHI} \text { of liver and lung } \mathrm{M} \phi \\
\mathrm{PHI} \text { of liver and lung } \mathrm{M} \phi \\
\mathrm{PHI} \text { of liver and lungM } \phi\end{array}$ & $\begin{array}{c}\uparrow \\
\uparrow \\
\uparrow \\
\uparrow \\
\uparrow\end{array}$ \\
\hline
\end{tabular}

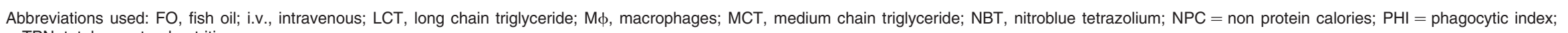
TPN, total parenteral nutrition. 


\begin{tabular}{|c|c|c|c|c|c|c|c|}
\hline Reference & Study in & Type of LE & Via & Dose & $\begin{array}{l}\text { Time } \\
\text { (h) }\end{array}$ & Function & Findings \\
\hline Waitzberg et al. 1992 & $\begin{array}{l}\text { Rats with E. coli } \\
\text { Periotonitis }\end{array}$ & $\begin{array}{l}\text { LCT } 10 \% \\
\text { MCT/LCT } 10 \% \\
\text { MCT } 10 \%\end{array}$ & i.v. & $0.5 \mathrm{ml} / \mathrm{kg}$ per $\mathrm{h}$ & 44 & $\begin{array}{l}\text { Chemotaxis } \\
\text { Phagocytosis } \\
\text { Bacterial killing }\end{array}$ & $\begin{array}{l}\text { No alteration } \\
\text { No alteration } \\
\text { No alteration }\end{array}$ \\
\hline Waitzberg et al. 1996 & Rats & $\begin{array}{l}\text { LCT } 10 \% \\
\text { MCT/LCT } 10 \%\end{array}$ & i.v. & $\begin{array}{l}1 \mathrm{ml} / \mathrm{h} \\
1 \text { or } 1.5 \mathrm{ml} / \mathrm{h}\end{array}$ & $\begin{array}{l}30 \\
44\end{array}$ & $\begin{array}{l}\text { Phagocytosis } \\
\text { Chemotaxis }\end{array}$ & $\begin{array}{l}\text { No alteration } \\
\text { No alteration }\end{array}$ \\
\hline & Rats & $\begin{array}{l}\text { LCT } 10 \% \\
\text { MCT } 10 \% \\
\text { MCT/LCT } 10 \%\end{array}$ & i.v. & $20 \mathrm{mg} / \mathrm{ml}$ & 0.5 & $\begin{array}{l}\text { Phagocytosis } \\
\text { Bacterial killing } \\
\text { Chemotaxis }\end{array}$ & $\begin{array}{l}\downarrow(\mathrm{LCT} \& \mathrm{MCT} / \mathrm{LCT}) \\
\downarrow(\mathrm{MCT}) \\
\downarrow(\mathrm{MCT})\end{array}$ \\
\hline Jarstrand et al. 1978 & Healthy volunteers & LCT $20 \%$ & i.v. & $20-100 \mathrm{mg} / \mathrm{ml}$ & 0,5 & $\begin{array}{l}\text { NBT reduction } \\
\text { Bacterial killing }\end{array}$ & $\downarrow$ \\
\hline & Patients (8) & LCT $20 \%$ & i.v. & $100 \mathrm{ml} / \mathrm{h}$ & 1 & NBT reduction & $\downarrow$ \\
\hline & Patients (6) & LCT $20 \%$ & i.v. & $100 \mathrm{ml} / \mathrm{h}$ & 1 & Bacterial killing & $\downarrow$ \\
\hline $\begin{array}{l}\text { Nordenstrom et al. } \\
1979\end{array}$ & Healthy volunteers (12) & LCT $20 \%$ & i.v. & $50-200 \mathrm{ml} / \mathrm{h}$ & 2 & Chemotaxis & $\downarrow$ (dose dependent) \\
\hline Fischer et al. 1980 & Healthy volunteers & LCT & i.v. & $12.5-100 \mathrm{mg} / \mathrm{ml}$ & 0.5 & Chemotaxis & $\downarrow$ \\
\hline English et al. 1981 & Healthy volunteers & LCT $10 \%$ & i.v. & $80 \mathrm{mg} / \mathrm{ml}$ & 0.5 & $\begin{array}{l}\text { Chemotaxis } \\
\text { Superoxide production } \\
\text { Chemiluminescence }\end{array}$ & $\begin{array}{l}\downarrow \text { if LCT not dialysed } \\
\downarrow \text { if LCT not dialysed } \\
\downarrow \text { if LCT not dialysed } \\
\text { No alteration if LCT dialysed against } \\
\text { saline }\end{array}$ \\
\hline Palmblad et al. 1982 & $\begin{array}{l}\text { Crohn's Disease (4) } \\
\text { Healthy volunteers (10) }\end{array}$ & $\begin{array}{l}\text { LCT } 20 \% \\
\text { LCT } 20 \%\end{array}$ & $\begin{array}{l}\text { i.v. } \\
\text { i.v. }\end{array}$ & $\begin{array}{l}21 \mathrm{ml} / \mathrm{h} \\
85 \mathrm{ml} / \mathrm{h}\end{array}$ & $\begin{array}{r}10 \\
6\end{array}$ & $\begin{array}{l}\text { Bacterial killing } \\
\text { Chemiluminescence } \\
\text { Chemotaxis }\end{array}$ & $\begin{array}{l}\text { No alteration } \\
\text { No alteration } \\
\uparrow\end{array}$ \\
\hline Wiernik et al. 1983 & Healthy volunteers (18) & LCT $20 \%$ & i.v. & $20-100 \mathrm{mg} / \mathrm{ml}$ & 0.5 & $\begin{array}{l}\text { NBT reduction } \\
\text { Chemotaxis }\end{array}$ & $\downarrow$ \\
\hline Cleary et al. 1983 & Healthy volunteers & LCT10\% & i.v. & $15-100 \mathrm{mg} / \mathrm{ml}$ & 0.5 & $\begin{array}{l}\text { NBT reduction } \\
\mathrm{O}_{2} \text { consumption } \\
\text { Bacterial killing } \\
\text { Phagocytosis }\end{array}$ & $\begin{array}{l}\uparrow \text { (dose dependent) } \\
\uparrow \text { (dose dependent) } \\
\uparrow \text { (dose dependent) } \\
\uparrow \text { (dose dependent) } \\
\text { But } \downarrow \text { of all functions with high dose }\end{array}$ \\
\hline Ota et al. 1985 & Cancer patients (40) & $\begin{array}{l}\text { LCT } 20 \% \\
(+ \text { TPN) }\end{array}$ & i.v. & $500 \mathrm{ml} /$ day & 240 & Phagocytosis & No alteration \\
\hline & & & & & & $\begin{array}{l}\text { Chemotaxis } \\
\text { Bacterial killing }\end{array}$ & $\begin{array}{l}\text { No alteration } \\
\text { No alteration }\end{array}$ \\
\hline Escudier et al. 1986 & $\begin{array}{l}\text { Surgical cancer patients } \\
\text { (9) }\end{array}$ & $\begin{array}{l}\text { LCT } 20 \% \\
(+ \text { TPN })\end{array}$ & i.v. & $83 \mathrm{ml} / \mathrm{h}$ & 6 & Chemotaxis & No alteration \\
\hline Rasmussen et al. 1988 & $\begin{array}{l}\text { Minor surgical patients } \\
\text { (24) }\end{array}$ & LCT $20 \%$ & i.v. & $100 \mathrm{ml} / \mathrm{h}$ & 4 & Opsonization time & No alteration \\
\hline & & & & & & $\begin{array}{l}\mathrm{O}_{2} \text { consumption } \\
\text { Superoxide production } \\
\text { Phagocytosis } \\
\text { Candida albicans killing } \\
\text { Chemotaxis }\end{array}$ & $\begin{array}{l}\text { No alteration } \\
\text { No alteration } \\
\text { No alteration } \\
\text { No alteration } \\
\text { No alteration }\end{array}$ \\
\hline Monico et al. 1988 & Healthy volunteers (40) & MCT/LCT $10 \%$ & i.v. & $120 \mathrm{ml} / \mathrm{h}$ & $5-7$ & $\begin{array}{l}\text { Chemotaxis } \\
\text { Adherence to nylon } \\
\text { fibers } \\
\text { Phagocytosis }\end{array}$ & $\begin{array}{l}\text { No alteration } \\
\text { No alteration } \\
\text { No alteration }\end{array}$ \\
\hline Robin et al. 1989 & $\begin{array}{l}\text { Patients (25) } \\
\text { Healthy volunteers (10) }\end{array}$ & $\begin{array}{l}\text { LCT10 \% } \\
\text { LCT } 10 \%\end{array}$ & $\begin{array}{l}\text { i.v. } \\
\text { i.v. }\end{array}$ & $\begin{array}{l}100 \mathrm{ml} / \mathrm{h} \\
400 \mathrm{mg} / \mathrm{l}\end{array}$ & $\begin{array}{l}4-6 \\
0.75\end{array}$ & $\begin{array}{l}\text { Chemiluminescence } \\
\text { Chemiluminescence }\end{array}$ & $\stackrel{\downarrow}{\text { No alteration }}$ \\
\hline Herson et al. 1989 & III neonates (10) & LCT & i.v. & $1 \mathrm{~g} / \mathrm{kg}$ & 16 & Chemotaxis & No alteration \\
\hline
\end{tabular}


Table 2. Continued

\begin{tabular}{|c|c|c|c|c|c|c|c|}
\hline Reference & Study in & Type of LE & Via & Dose & $\begin{array}{l}\text { Time } \\
\text { (h) }\end{array}$ & Function & Findings \\
\hline \multirow{2}{*}{$\begin{array}{l}\text { Bellinatti-Pires et al. } \\
1992\end{array}$} & Healthy volunteers & LCT $10 \%$ & $\begin{array}{l}\text { i.v. } \\
\text { i.v. }\end{array}$ & $\begin{array}{l}100 \mathrm{mg} / \mathrm{ml} \\
20 \mathrm{mg} / \mathrm{ml}\end{array}$ & $\begin{array}{l}0.5 \\
0.5\end{array}$ & $\begin{array}{l}\text { Chemotaxis } \\
\text { Chemotaxis }\end{array}$ & $\begin{array}{l}\text { No alteration } \\
\downarrow \text { (dose dependent) in MCT groups }\end{array}$ \\
\hline & & $\begin{array}{l}\text { MCT } 10 \% \\
\text { MCT/LCT } 10 \%\end{array}$ & & & & & \\
\hline \multirow{2}{*}{$\begin{array}{l}\text { Bellinatti-Pires et al. } \\
1993\end{array}$} & Healthy volunteers (20) & LCT $10 \%$ & i.v. & $20 \mathrm{mg} / \mathrm{ml}$ & 0.5 & NBT reduction & $\downarrow$ in MCT groups \\
\hline & & $\begin{array}{l}\text { MCT } 10 \% \\
\text { MCT/LCT } 10 \%\end{array}$ & & & & $\begin{array}{l}\text { Phagocytosis } \\
\mathrm{H}_{2} \mathrm{O}_{2} \text { production } \\
\text { Bacterial killing }\end{array}$ & $\begin{array}{l}\downarrow \text { in MCT groups } \\
\downarrow \text { in MCT groups } \\
\downarrow \text { in MCT groups }\end{array}$ \\
\hline \multirow[t]{2}{*}{ Waitzberg et al. 1997} & $\begin{array}{l}\text { Gastric cancer patients } \\
\text { (10) }\end{array}$ & LCT $10 \%$ & i.v. & $\begin{array}{l}10 \mathrm{kcal} / \mathrm{d}(0.08 \mathrm{~g} / \mathrm{kg} \text { per } \\
\mathrm{h})\end{array}$ & 48 & Chemotaxis & No alteration \\
\hline & & $\begin{array}{l}\text { MCT/LCT } 10 \% \\
(+ \text { TPN })\end{array}$ & & & & $\begin{array}{l}\text { NBT reduction } \\
\text { Bacterial killing }\end{array}$ & $\begin{array}{l}\text { No alteration } \\
\downarrow\end{array}$ \\
\hline Heine et al. 1999 & Healthy volunteers (10) & $\begin{array}{l}\text { LCT } \\
\text { MCT/LCT } \\
\text { LCT with FO }\end{array}$ & i.v. & $60-600 \mathrm{mg} / \mathrm{ml}$ & $<0.5$ & Respiratory burst & $\begin{array}{l}\downarrow \\
\downarrow \\
\uparrow \\
\downarrow\end{array}$ \\
\hline Kruimel et al. 2000 & Healthy volunteers & $\begin{array}{l}\mathrm{LCT} \\
\mathrm{MCT} / \mathrm{LCT} \\
\mathrm{MCT} / \mathrm{LCT} \\
\text { structured }\end{array}$ & i.v. & $1-100 \mathrm{mmol} / \mathrm{l}$ & 0.5 & $\begin{array}{l}\text { Chemiluminescence } \\
\text { ROS production }\end{array}$ & $\begin{array}{l}\uparrow \text { with MCT/LCT } \\
\uparrow \text { rate and amount with } \\
\text { MCT/ILCT }\end{array}$ \\
\hline
\end{tabular}

Abbreviations used: FO, fish oil; i.v., intravenous; LCT, long chain triglyceride; MCT, medium chain triglyceride; NBT, nitroblue tetrazolium; TPN, total parenteral nutrition. 
monocytes was noted. Preincubation of monocytes in vitro with LCT LE $(20-100 \mathrm{mg} / \mathrm{ml})$ for $30 \mathrm{~min}$ was found to increase the ability of the cells to migrate chemotactically and to phagocytose yeast particles.

In a prospective, randomized, cross-over clinical trial malnourished gastric cancer patients received a $48 \mathrm{~h}$ TPN $(40 \mathrm{kcal} / \mathrm{kg}$ ) with $25 \%$ of energy provided as LCT or MCT/LCT LE (infused at the rate of $0.08 \mathrm{~g} / \mathrm{kg}$ per h). No alteration in monocyte-macrophage function and phagocytosis was found with either LE compared to the appropriate controls (Waitzberg et al. 1997).

\section{Effects of lipid emulsions on neutrophils}

Neutrophils are cells related to natural immunity. They represent the first cellular defense against microorganisms. Their initial functions are to phagocytose and inactivate invasive agents. Despite numerous reports about interactions between lipids and the phagocytic system, the effects of LE on the metabolic and microbial killing activities of neutrophils are still controversial (Table 2).

\section{Experimental studies}

Experimental data indicate an inhibitory effect of LCT and MCT/LCT LE on rat neutrophil phagocytosis in vitro (Waitzberg et al. 1996), but no differences in ex vivo phagocytosis, chemotaxis and bacterial killing were observed in healthy and septic rats when LCT LE, MCT/LCT LE or saline were intravenously administrated (Waitzberg et al. 1992, 1996).

From the in vitro studies performed using human polymorphonuclear cells a dose dependent response to LE is apparent. For instance, soybean oil emulsions showed an inhibitory effect on neutrophils when used at concentrations ranging from $15 \mathrm{mg} / \mathrm{ml}$ to $100 \mathrm{mg} / \mathrm{ml}$ but when concentrations higher than $100 \mathrm{mg} / \mathrm{ml}$ were used, no alterations in neutrophil functions were found (Jarstrand et al. 1978; Wiernik et al. 1983; English et al. 1981; Bellinati-Pires et al. 1992). Moreover, with MCT/LCT LE a stimulatory effect was documented (Robin et al. 1989; Bellinati-Pires et al. 1992; Bellinati-Pires et al. 1993; Heine et al. 1999), when low doses were used.

In clinical studies, the capacity of neutrophils to phagocytose was not altered with LCT LE (Ota et al. 1985; Rasmussen et al. 1988), or MCT/LCT (Monico et al. 1988; Dominioni \& Dionigi, 1987). Chemotaxis was also unchanged in five studies with LCT-LE (Ota et al. 1985; Rasmussen et al. 1988; Escudier et al. 1986; Herson et al. 1989; Waitzberg et al. 1997). However, a dose-dependent inhibitory effect was detected in a single study (Nordenstrom et al. 1979) and also the opposite, a stimulatory effect, has been demonstrated. (Palmblad et al. 1982). Bacterial killing by polymorphonuclear neutrophils (PMN) is controversial: three studies did not find any alteration (Ota et al. 1985; Palmblad et al. 1982; Rasmussen et al. 1988) and two studies showed an inhibition with LCT LE (Jarstrand et al. 1978; Waitzberg et al. 1997). In a clinical trial with gastric cancer patients receiving TPN with LCT or MCT/LCT LE, bacterial killing by neutrophils was the only function reduced after LCT LE, although this function remained within the normal range values in $80 \%$ of the patients. In conclusion, LCT LE moderately decreases neutrophil bacterial killing.

There is little literature involving nitroblue-tetrazolium (NBT) reduction and superoxide anion production.Two studies showed no change (Rasmussen et al. 1988; Waitzberg et al. 1997) and one study an inhibitory effect (Jarstrand et al. 1978).

There are no data available regarding the effect of $n-3$ fatty acid-containing LE or olive oil emulsion on neutrophil function.

\section{Potential immunomodulatory mechanisms of lipid emulsions}

In the last years, several mechanisms have been proposed to explain the different effects of LE on the immune system. They are associated mainly with fatty acid incorporation and modification of cell membrane composition (Palombo et al. 1997).

The effects of fatty acids on phagocytic activity in vivo and in vitro are controversial. The presence of dietary polyunsaturated fatty acids may inhibit microbicidal activity. However, polyunsaturated fatty acids may stimulate this activity when directly added to cell culture. One possible explanation for the controversial results is provided by Mahoney et al. $(1977,1980)$. These authors calculated that, in normal conditions, the phagocytosis of an erythrocyte results in approximately $7 \%$ internalization of the macrophage surface area.

Membrane fatty acid composition influences membrane fluidity, cell permeability, eicosanoid production, as well as membrane receptors, enzymes and second messenger signaling processes. Although $n-6$ and $n-3$ polyunsaturated fatty acids are incorporated into cell membranes, it remains to be proved if medium chain triglycerides or saturated fatty acids are also incorporated into cell membranes when present in doses higher than in a normal diet. Fatty acids may interfere with other immune modulating mechanisms such as modulation of adhesion molecules, regulation of nitric oxide (NO) production, modulation of signal transduction pathways and direct modulation of gene expression (Yaqoob, 1998).

Enzymes such as lipoxygenases and cyclooxygenases produce proinflammatory lipid mediators such as leukotriene (LT) $\mathrm{B}_{4}$ and prostaglandin $(\mathrm{PG}) \mathrm{E}_{2}$ from arachidonic acid. Several eicosanoids such as $\mathrm{LTB}_{4}$ and thromboxane $\mathrm{B}_{2}$ $\left(\mathrm{TXB}_{2}\right)$ have chemotactic effects. When exposed to higher concentrations of $n-3$ polyunsaturated fatty acids, lipoxygenases and cyclooxygenases produce less biologically active mediators as $\mathrm{LTB}_{5}$ and $\mathrm{PGE}_{3}$ (Magrum \& Johnston, 1983). An n-3 fatty acid rich diet lowers $\mathrm{PGE}_{2}$, 6-keto$\mathrm{PGF}_{1}$-alpha, $\mathrm{LTB}_{4}$ and $\mathrm{TXB}_{2}$ production when compared to an $n-6$ fatty acid rich diet. $n-6$ Fatty acids augment $\mathrm{PGE}_{2}$, $\mathrm{LTB}_{4}, \mathrm{LTC}_{4}, \mathrm{LTD}_{4}$, 6-keto-PGF 1 -alpha and $\mathrm{TXB}_{2}$, probably via arachidonic acid, the eicosanoid precursor.

Membrane phospholipids can be transformed by phospholipases to yield second messengers (such as phosphatidylinositol-4,5-bisphosphate generating diacylglycerol and inositol-1,4,5-trisphosphate) and can be involved in activating or stabilizing enzymes in signaling pathways 
(such as phophatidylserine that is required for the activation of protein kinase C). All phospholipids and some of the second messengers that they generate contain fatty acyl chains, and it is possible that changes in membrane fatty acid composition may alter properties of these compounds. (Serhan et al. 1996; Ballou et al. 1996; Yaqoob, 1998).

Recent evidence shows that lipids are direct modulators of gene expression. Therefore, changes in cellular fatty acid profiles or in fatty acid metabolites could affect the activity of specific transcription factors, which alter gene transcription (indirect modulation of gene expression). The peroxisome proliferator-activated receptor family (PPAR - a group of key nuclear receptors involved in the regulation of lipid homeostasis) regulate the expression of target genes by binding to DNA sequence elements. It has been shown that several unsaturated fatty acids bind directly to PPARalpha (Yaqoob, 1998). Through these actions fatty acids could regulate expression of adhesion proteins, such as integrins and selectins, thus altering cell adhesion.

NADPH oxidase is an enzyme present in neutrophils which produces free radicals and may be stimulated by arachidonic acid. Diets enriched with $n-6$ or saturated fatty acids are associated with decreased peroxide and free radical production by activated neutrophils and to down regulation of glucose-6-phosphate-dehydrogenase. The latter is one of the most powerful inducers of NADPH synthesis, and an essential substrate for free radical production by NADPH oxidase. NO production is catalysed in phagocytic cells by NO synthetase induction. Transcription of NO synthetase and NO production may be inhibited by polyunsaturated fatty acids.

Despite the different immunomodulating properties of fatty acids it should be kept in mind that LE for parenteral use are complex nutrient sources composed not only of fatty acids but also including phosphatidylcholine, glycerol, and $\alpha$-tocopherol. Besides that, LE contain various types of triglycerides in a physical or structured mixture. The interaction of these distinct substances may alter the effects observed with isolated fatty acids (Kruimel et al. 2000; De la Fuente, 2000).

\section{Clinical impact and conclusions}

In clinical practice, LE for parenteral use have been employed for more than 30 years. The inhibitory effect of LE on the functional activity of the phagocytic system, although still controversial, may be an important harmful impact because TPN with lipids may be recommended in hypercatabolic conditions where inflammation and infection are present.

LCT LE are still the main parenteral fat source and are rich in $n-6$ fatty acids. The concerns about the association of LE with infectious morbidity were raised after a report of an increased risk of bacteriemia related to the use of LE in neonates (Freeman et al. 1990). However in an extensive clinical trial in patients undergoing bone marrow transplantation while receiving TPN there was no evidence that moderate doses of a LCT LE were associated with the incidence of bacterial or fungal infections (Lenssen et al. 1998).

LCT LE may have adverse effects on the immune system, especially when they are given in high doses over a short period of time. However, when administered properly they can be used safely for clinical purposes (Klein \& Miles, 1994).

MCT containing LE, in a physical or structured way, may bring some advantages because of their positive effects on polymorphonuclear cells, macrophages, and cytokine production, particularly in critically ill or immunocompromized patients. This is one of the reasons to consider substitution of LCT LE with MCT/LCT LE. Accordingly, there is a tendency to prefer MCT/LCT emulsions in critically ill patients before and after surgery and in sepsis. LCT LE should be limited in specific clinical conditions like burns, cancer, hepatic dysfunction, immunocompromized status and metabolic stress.

On the other hand, fish oil containing LE, may have some advantages in patients with autoimmune diseases such as rheumatoid arthritis and intestinal inflammatory diseases or in special clinical situations where an anti-inflammatory activity is desirable (Calder, 1998).

In spite of the optimistic preliminary results concerning the parenteral use of olive oil, its effects need to be confirmed by more prospective, randomized clinical trials. Variables like amount, and type of fatty acids, infusion rates and TPN duration should also be considered.

New parenteral lipid emulsions containing $n-3$ polyunsaturated fatty acids and monounsaturated olive oil are already available in Europe. Judicious use of these new LE is mandatory especially relating to the impact on the immune system. New experimental and clinical studies are required to further establish the role of $\mathrm{LE}$ in clinical nutrition.

\section{References}

Allen TM \& Murray L (1986) Effect of long-term intralipid administration in mice. Canadian Journal Physiology and Pharmacology 64, 1006-1010.

Ballou LR, Laulederkind SJ, Rosloniec EF \& Raghow R (1996) Ceramide signalling and the immune response. Biochimica Biophysica Acta 1301, 273-287.

Beisel WR (1981) Impact of infectious disease upon fat metabolism and immune functions. Cancer Research 41, 3797-3798.

Bellinati-Pires R, Waitzberg DL, Salgado MM \& CarneiroSampaio MM (1992) Effect of medium - and long-chain triglycerides on human neutrophil migration. Brazilian Journal Medical and Biology Research 25, 369-373.

Bellinati-Pires R, Waitzberg DL, Salgado MM \& CarneiroSampaio MM (1993) Functional alterations of human neutrophils by medium-chain triglyceride emulsions: evaluation of phagocytosis, bacterial killing, and oxidative activity. Journal of Leukocyte Biology 53, 404-410.

Calder PC (1998) Immunoregulatory and anti-inflammatory effects of n-3 polyunsaturated fatty acids. Brazilian Journal Medical and Biology Research 31, 467-490.

Chandra RK (1985) Trace element regulation of immunity and infection. Journal of the American College of Nutrition 4, 5-16.

Cleary TG \& Pickering LK (1983) Mechanisms of intralipid effect on polymorphonuclear leukocytes. Journal of Clinical and Laboratorial Immunology 11, 21-26.

Cukier C, Waitzberg DL, Logullo A, Bacchi VH, Torrinhas RS, Soares RS, Saldiva PH, Oliveiram TS \& Heymsfield S (1999) 
Lipid and lipid-free total parenteral nutrition: differential effects on macrophage phagocytosis in rats. Nutrition 15, 885-889.

De la Fuente M, Carazo M, Correa R \& Del Rio M (2000) Changes in macrophage and lymphocyte function in guinea-pigs after different amounts of vitamin E ingestion. British Journal of Nutrition 84, 25-29.

DeWille JW, Fraker PJ \& Romsos DR (1979) Effect of essential fatty acid deficiency, and various levels of polyunsaturated fatty acids and humoral immunity in mice. Journal of Nutrition 109, $1018-1027$.

DiLuzio NR (1972) Employment of lipids in the measurement and modification of cellular, humoral and immune responses. In Advances in Lipid Research, pp. 43-48 [R Paoletti and D Kritchevsky, editors]. New York, NY: Academic Press.

Dominioni L \& Dionigi R (1987) Immunological function and nutritional assessment. Journal of Parenteral and Enteral Nutrition 11(5 Suppl), 70S-72S.

Dudrick SJ, Wilmore DW, Vars HM \& Rhoads JE (1969) Can intravenous feeding as the sole means of nutrition support growth in the child and restore weight loss in an adult? An affirmative answer. Annals of Surgery 169, 974-984.

English D, Roloff JS, Lukens JN, Greene HL \& Ghishan FK (1981) Intravenous lipid emulsions and human neutrophil functions. Journal of Pediatrics 99, 913-916.

Escudier EF, Escudier BJ, Henry-Amar MC, Lobut JB, Bernaudin F, Leclerq BP, Netenberg GM \& Bernauden JF (1986) Effects if infused intralipid on neutrophil chemotaxis during total parenteral nutrition. Journal of Parenteral and Enteral Nutrition 10, 596-598.

Fischer GW, Hunter KW, Wilson SR \& Mease AD (1980) Diminished bacterial defenses with intralipid. Lancet 18, 819-820.

Freeman J, Goldmann DA, Smith NE, Sidebottom DG, Epstein MF \& Platt R (1990) Association of intravenous lipid emulsion and coagulase-negative staphylococcal bacteremia in neonatal intensive care units. New England Journal of Medicine 323, $301-308$.

Hamawy KJ, Moldawer LL, Georgiff M, Valicente Aj, Babayan VK, Bistrian BR \& Blackburn GL (1985) The effect of lipid emulsions on reticuloendothelial system function in the injured animal. Journal of Parenteral and Enteral Nutrition 9, $559-565$.

Heine J, Scheinichen D, Jaeger K, Andre M \& Leuwer M (1999) In vitro influence of parenteral lipid emulsions on the respiratory burst of neutrophils. Nutrition 15, 540-545.

Herson VC, Block C, Eisenfeld L, Maderazo EG \& Krause PJ (1989) Effects of intravenous fat infusion on neonatal neutrophil and platelet function. Journal of Parenteral and Enteral Nutrition 13, 620-622.

Hwang D (1989) Essential fatty acids and the immune response. Federation of American Societies of Experimental Biology Journal 3, 2052-2061.

Jarstrand C, Berghem L \& Lahnborg G (1978) Human granulocyte and reticuloendothelial system function during intralipid infusion. Journal of Parenteral and Enteral Nutrition 2, $663-670$.

Kinsella JE (1990) Lipids, membrane receptors, and enzymes: effects of dietary fatty acids. Journal of Parenteral and Enteral Nutrition 14, 200S-217S.

Klein S \& Miles JM (1994) Metabolic effects of long chain and medium chain triglyceride emulsions in humans. Journal of Parenteral and Enteral Nutrition 18, 396-397.

Koss WL, Loria RM, Snodgrass MJ, Cohen D, Thorpe TG \& Kaplan AM (1979) Inhibition of host resistance by nutritional hypercholesterolemia. Infectious Immunology 26, 658-667.

Kruimel JW, Naber AH, Curfs JH, Wenker MA \& Jansen JB (2000) With medium-chain triglycerides, higher and faster oxygen radical production by stimulated polymorphonuclear leukocytes occurs. Journal of Parenteral and Enteral Nutrition 24, 107-112.

Lenssen P, Bruemmer BA, Bowden RA, Gooley T, Aker SN \& Mattson D (1998) Intravenous lipid dose and incidence of bacteremia and fungemia in patients undergoing bone marrow tranplantation. American Journal of Clinical Nutrition 67, 927-933.

Magrum LJ \& Johnston PV (1983) Modulation of prostaglandin synthesis in rat peritoneal macrophages with w-3 fatty acids. Lipids 18, 514-521.

Mahoney EM, Hamill AL, Scott WA \& Cohn ZA (1977) Response of endocytosis to altered fatty acyl composition of macrophage phospholipids. Proceedings of the National Academy of Sciences of USA 74, 4895-4899.

Mahoney EM, Scott WA, Landsberger FR, Hamill AL \& Cohn ZA (1980) Influence of fatty acyl substitution on the composition and function of macrophage membranes. Journal of Biology and Chemistry 255, 4910-4917.

Meade CJ \& Mertin J (1978) Fatty acids and immunity. Advances in Lipid Research 16, 127-165.

Mertin J \& Meade CJ (1977) Relevance of fatty acids to multiple sclerosis. British Medical Bulletin 33, 67-71.

Monico R, Dominioni L, Interdonato L, Festi L \& Dionigi R (1988) Effects of IV administration of MCT containing fat emulsion on neutrophil function and chemistry. In Beitrage zur Klin Infusionstherapie, p. 36 [W Creutzveld and P Schauder, editors]. Basel: Karger.

Mullen JL, Buzby GP, Matthews DC, Smale BF \& Rosati EF (1980) Reduction of operative morbidity and mortality by combined preoperative and postoperative nutritional support. Annals of Surgery 192, 604-613.

Nishiwaki H, Iriyama K, Asami H, Kihata M, Hioki T, Asakawa T \& Suzuki H (1986) Influences of an infusion of lipid emulsion on phagocytotic activity of cultured Kupffer's cells in septic rats. Journal of Parenteral and Enteral Nutrition 10, 614-616.

Nordenstrom J, Jarstrand C \& Wiernick A (1979) Decreased chemotactic and random migration of leukocytes during intralipid infusion. American Journal of Clinical Nutrition 32, 2416-2422.

Nugent KM (1984) Intralipid effects on reticuloendothelial function. Journal of Leukocyte Biology 36, 123-132.

Ota DM, Jessup JM, Babcock GF, Kirschbaum R, Mountain CF, Mc Murtrey MJ \& Copeland EM (1985) Immune function during intravenous administration of a soybean oil emulsion. Journal of Parenteral and Enteral Nutrition 9, 23-27.

Palombo JD, De Michele SJ, Lydon EE \& Bistrian BR (1997) Cyclic vs. Continuous enteral feeding with omega-3 and gamma linolenic fatty acids: effects on modulation of phospholipid fatty acids in rat lung and liver immune cells. Journal of Parenteral and Enteral Nutrition 21, 123-132.

Palmblad J, Brostrom O, Lahnborg G, Uden AM \& Venizelos N (1982) Neutrophil functions during total parenteral nutrition and Intralipid infusion. American Journal of Clinical Nutrition 35, 1430-1436.

Piette M \& Saugier J (1970) Effect of the intravenous infusion of a lipid emulsion on blood leukocytes in the rabbit. Annals Pharmacie Francese 28, 529-534.

Rasmussen A, Hessov I \& Segel E (1988) The effect of Intralipid on polymorphonuclear leukocytes. Clinical Nutrition 7, 37-43.

Robin AP, Arain I, Phuangsab A, Holian O, Roccaforte P \& Barrett JA (1989) Intravenous fat emulsions acutely suppresses neutrophil chemiluminescence. Journal of Parenteral and Enteral Nutrition 13, 608-614.

Serhan CN, Haeggström JZ \& Leslie CC (1996) Lipid mediator networks in cell signalling: update and impact of cytokines. 
Federation of American Societies of Experimental Biology Journal 10, 1147-1158.

Shils ME (1998) Parenteral nutrition. In Modern Nutrition in Health and Disease, Ninth edition, [ME Shils, JA Olson, ME Shike and C Ross, editors]. Baltimore, PA: Williams \& Wilkins.

Shou J, Lappin J, Minnard EA \& Daly JM (1994) Total parenteral nutrition, bacterial translocation, and host immune response. American Journal of Surgery 167, 145-150.

Sobrado J, Moldawer LL, Pomposelli JJ, Mascioli EA, Babayan VK, Bistrian BR \& Blackburn GL (1985) Lipid emulsions and reticuloendothelial system function in healthy and burned guinea pigs. American Journal of Clinical Nutrition 42, $855-863$.

Strunk RC, Payne CM, Nagle RB \& Kunke K (1979) Alteration of the structure and function of guinea pig peritoneal macrophages by a soybean oil emulsion. American Journal of Pathology 96, $753-770$.

Vallgren M, Tranberg KG, Vallgre S, Bjornson HS \& Fischer JE (1986) Effect of intralipids on clearance and organ uptake of bacteria in intra-abdominal sepsis rat. Clinical Nutrition $\mathbf{5}$, $7-11$.

Waitzberg DL, Bellinati-Pires R, Salgado MM, Hypolito IP, Colleto GM, Yagi O \& Yamamuro EM (1997) Effect of total parenteral nutrition with different lipid emulsions on human monocyte and neutrophil functions. Nutrition 13, 128-132.

Waitzberg DL, Yamaguchi N, Bellinati-Pires R, Leone MC, Masili-Oku SM, Salgado MM, Soares SR \& Lima-Gonçalves EL (1992) Efeito de emulsões lipídicas sobre os mecanismos de defesa orgânica na agressão infecciosa. Revista do Hospital das Clinicas da Faculdade de Medicina de São Paulo 47, 215-222.

Waitzberg DL, Bellinati-Pires R, Yamaguchi N, Massili-Oku SM, Salgado MM, Hypolito IP, Soares SR, Gonçalves EL \& Furst P (1996) Influence of medium chain triglyceride-based lipid emulsion on rat polymorphonuclear cell functions. Nutrition $\mathbf{1 2}$, 93-99.

Wiernik A, Jarstrand C \& Julander I (1983) The effect of intralipid on mononuclear and polymorphonuclear phagocytes. American Journal of Clinical Nutrition 37, 256-261.

Yaqoob P (1998) Lipids and the immune response. Current Opinion Clinical Nutrition and Metabolic Care 1, 153-161. 